\title{
LA REUNIFICACION DE ALEMANIA EN EL MARCO DE LAS COMUNIDADES EUROPEAS *
}

La región de la antigua RDA (República Democrática Alemana) ${ }^{(1)}$ ha desempeñado desde el pincipio cierto rol especial en el derecho de las Comunidades Europeas. Este hecho se manifiesta especialmente en los protocolos (incorporados como apéndice a los "Tratados para la fundación de la Comunidad Europea") sobre el "comercio interno alemán", sobre el concepto de "nacionalidad alemana" y sobre la "validez de los tratados para Berlín". Además, los tratados fundacionales de la C.E. (Comunidad Europea) contienen ciertas excepciones, referidas a las consecuencias de la división de Alemania. La ex RDA ha sido siempre, en cierto sentido, también un "socio tácito" de la C.E. sin que por ello, a través de las correspondientes decisiones, se hubiera consolidado el "statu quo".

Después de la caída del muro de Berlín ( 8 de noviembre de 1.989) se planteó de inmediato el problema sobre la manera en que debería alcanzarse la reunificación de Alemania. La Constitución de Alemania Occidental ${ }^{(2)}$, en vigencia desde 1.949 , contenía dos formas diferentes de solución. Por un lado, se trata de la posibilidad de ingreso de Alemania Oriental a la República Federal de Alemania en los términos del art. 23 de la Constitucion ${ }^{(3)}$. La segunda opción (art. 146 de la Constitución) consistió en la unificación de ambos Estados en Alemnania sobre la base de una nueva Constitución (4).

Para la integración del territorio de la antigua RDA en la C.E., el derecho comunitario dispuso de dos posibilidades, que se corresponden con ambas opciones de la Constitución germano occidental:

\footnotetext{
"Titulo original "Die Wiedervereinigung Deutschlands in Rahmen der Europaeischen Gemeinschaften" La versión castellana corresponde al Profesor Lic. Raúl Oscar Dichiara. Universidad Nacional del SurCONICET.

1) República Democrática Alemana (es decir, Alemania Oriental)

2) Es decir, la llamada "Ley Fundamental" de la República Federal de Alemania

3) Según el art. 23 de la Constitución esta ley es "aplicable a otras partes de Alemania... después de su ingreso".

4) Art. 146 de la Constitución establece: "Esta Ley Fundamental pierde su validez el día en que entre en vigencia una Constitución que haya sido acordada en libre decisión por los pueblos alemanes".
} 
art. 227 del Tratado de la Comunidad Económica Europea (5) o bien el art. 237 del mismo tratado. La alternativa mencionada en primer término es la incorporación automática de Alemania Oriental en la C.E. como una parte adicional de un Estado miembro (ampliado territorialmente), es decir, la República Federal de Alemania. Un procedimiento semejante requiere sólo el consenso de todos los Estados miembro de la C.E.; entonces, no se exige una aprobación formal de los socios de la C.E. porque con el ingreso de un Estado en otro, según el art. 23 de la Constitución, no se crearía un nuevo sujeto de derecho internacional ${ }^{(6)}$. La alternativa del art. 237 del Tratado de la Comunidad Económica Europea se aplicará en el caso de una reunificación según el art. 146 de la Constitución. Esto hubiera tenido como consecuencia, en principio, una separación de Alemania Occidental de la C.E.; la admisión de Alemania reunificada como nuevo Estado miembro (es decir, como un nuevo sujeto de derecho internacional) hubiera sido posible entonces, a partir de la entrada en vigencia de la nueva Constitución, sobre la base de un procedimiento regular. Este camino, que es habitual en casos normales, hubiera demorado varios años según la experiencia. En efecto, un procedimiento semejante se perfecciona en base a un acuerdo internacional especial, el que adquiere validez sólo cuando ha sido ratificado por todos los Estados miembro.

La discusión sobre cuál de ambos caminos debía ser elegido para la reunificación fue decidida relativamente rápido. Tanto en el ámbito de la C.E. cuanto también en Alemania se impuso -en vista de la situación política mundial- la concepción de que una solución según el art. 227 del Tratado de la Comunidad Económica Europea (y, consecuentemente, según el art. 23 de la Constitución) tendría las mayores ventajas. El 22 de diciembre de 1.989 la Comisión de la C.E. recibió mandato del Consejo de la C.E. para iniciar negociaciones con la RDA con el objeto de celebrar un Acuerdo no Preferencial de Comercio y Cooperación. Las negociaciones concluyeron en mayo de 1.990 con la firma de un acuerdo de esta naturaleza entre la C.E. y la RDA. Este contenía las mismas medidas de ayuda que pocos meses

5) Tratado para la fundación de la Comunidad Económica Europea.

6) Según el art. 23 de la Ley Fundamental, a través del ingreso de Alemania Oriental, la República Federal de Alemania subsiste inmodificada en terminos del derecho internacional, con excepción de sus fronteras externas. Según el art. 227 del Tratado de la Comunidad Económica Europea, la relevancia del mismo se refiere a todo el territorio nacional de un país miembro. Esto significa, que el derecho de la Comunidad Europea vigente es válido automáticamenie en las nuevas regiones de un país miembro ampliado (llamado "principio de las fronteras contractuales flexibles"). 
antes la C.E. había garantizado a Hungría y Polonia. Debido a la gran dinámica del desarrollo posterior, este acuerdo resultó de hecho obsoleto desde el principio. En la sesión especial de Jefes de gobierno y Estado de la C.E. en Dublin (21 de abril de 1.990) se alcanzó en principio consenso acerca de que el territorio de la RDA es integrado a la C.E. simultáneamente con su ingreso a la República Federal de Alemania.

\section{PROCESO DE INTEGRACION GRADUAL}

En la decisión de Dublin se acordó además que el orden jurídico, económico y social de la República Federal de Alemania y de la C.E. sólo puede aplicarse en los cinco (7) nuevos estados federales (i.e. en el territorio de la ex $\mathrm{RDA}$ ) paso a paso. En todas las ampliaciones anteriores de la C.E. la inclusión funcional de los nuevos miembros se realizó también en varios pasos sucesivos. La ampliación de la C.E. a los liamados cinco nuevos estados federales alemanes no ha sido por cierto un ingreso en el sentido del derecho internacional. No obstante, bajo aspectos económicos, este procedimiento es comparable a un ingreso regular. En vista de la admisión en la C.E. de nuevos países miembro ${ }^{(8)}$ verificadas en el pasado existen, en efecto, diferencias sustanciales. Así, las regiones que hasta ahora habían ingresado a la C.E., poseían al momento de su incorporación a la misma un orden establecido de economía de mercado. Además, dispusieron de más tiempo que Alemania Oriental para prepararse a ingresar a la C.E.

En abril de 1.990 se estableció un concepto en tres etapas para la integración fáctica en la C.E. de los cinco nuevos estados federales alemanes. La primera etapa comenzó el 1 de julio de 1.990 con la entrada en vigencia de la unión económica, monetaria y social germano-germano. Ese día se abolió la moneda germano-oriental y fue sustituída por el marco alemán occidental (DM). Simultáneamente, se procedió a los primeros pasos de la reforma del orden económico en Alemania Oriental. Entonces, paralelamente a la introducción del DM, se exigía también validez jurídica para una parte importante del derecho económico y social de la República Federal de Alemania en la, por aquel entonces aún existente, RDA.

La primera fase de la incorporación de Alemania Oriental concluyó con la unión formal de derecho público de ambos Estados

-) Brandeburgo: Mecklenburgo-Pomerania Anterior: Sajonia; Sajonia-Anhalt: Turingia.

8) 1.973: Gran Bretaña. Dinamarca. Irlanda; 1.981: Grecia; 1.986: España y Portuga! 
alemanes el día 3 de octubre de 1.990. La RDA desapareció así como sujeto de derecho intemacional. Simultáneamente, comenzó la segunda etapa de la integración de los cinco nuevos estados alemanes en la C.E. Esta segunda etapa es por su esencia una etapa de transición; está caracterizada por una serie de medidas de adaptación y excepciones transitorias a determinadas reglas. La última (tercera) etapa comenzará entonces cuando los cinco nuevos estados federales lleguen a pertenecer a la C.E. sin diferencia alguna en comparación con otro país/es miembros.

El período de transición, concebido como segunda etapa, comenzó formalmente como consecuencia del hecho de que el ingreso de la ex RDA a la República Federal de Alemania significó también la entrada en vigencia automática el 3 de octubre de 1.990 en los nuevos estadios federales del derecho primario y secundario de la C.E. (el llamado "acquis communautaire") ${ }^{(9)}$. En tanto que no fueron acordadas reglas especiales para la fase de transición, el acquis communautaire es de aplicación inmediata a partir de aquella fecha en la parte oriental de la Alemania unificada. Sin embargo, los socios de la C.E. acordaron desde el principio que, debido a las con secuencias económicas y sociales del proceso de conversión a la economía de mercado, existe una cierta necesidad transitoria de reglas de adaptación y excepción. También resultó claro que la iniciación de la aplicación de muchas prescripciones jurídicas de la C.E. debía ser postergada por determinado tiempo a causa de dificultades técnicas de reorganización.

Después de intensas negociaciones realizadas entre el gobierno federal alemán y las Comunidades Europeas apremiados por el tiempo, el Consejo de la C.E. aprobó con fuerza de ley el paquete íntegro de excepciones (10) transitorias el día 4 de diciembre de 1.990. Este resultado se alcanzó sólo después de haber garantizado que los productos de los nuevos estados federales, que aún no satisfacen las prescripciones de la C.E. (ejemplo: normas, especificaciones sobre medio ambiente y protección del consumidor) sólo pueden ser consumidos en el ámbito de estos estados. Análogo vale para la prosecución temporal de la importación libre de arancel por parte de los estados federales germano orientales desde los Estados del antiguo

9) La totalidad del derecho comunitario. que se ha originado en el transcurso de algo más de tres décadas, se designa habitualmen-te como "acquis communautaire". Este "fundamento societario" de la C.E. comprendia en 1.990 poco menos de 1.400 actas puridicas y su amplitud en el "Boletin Oficia! de la C.E." asciende a más de 12.000 páginas.

10) Publicado en el Boletin Oficial de la C.E.. L 353 del 1 de diciembre de 1.990, p. 1 y ss. 
COMECON. Los bienes que se correspondían con el derecho de la C.E. pudieron ser comercializados libremente en toda la C.E. de inmediato a partir del ingreso. De esta forma se estimuló adaptar la producción a los estándares de la C.E. tan rápido como sea posible.

Tal como se mencionó, los plazos acordados a un cierto número de sectores, en los que el derecho comunitario no es aún de aplicación, cumplen la función de una ayuda para la adaptación. Los diversos ámbitos de regulación pueden ser tratados aquí sólo a grandes rasgos $\mathrm{y}$ en casos puntuales.

Un elemento central de las regulaciones de transición concierne a los tratados comerciales que la antigua RDA había suscripto con sus anteriores socios del COMECON. El problema en este aspecto resulta de que estos tratados eran parte esencial del plan macroeconómico de los Estados del COMECON y, simultáneamente, la RDA dejó de ser sujeto de derecho internacional con el ingreso a la República Federal de Alemania. Con ello caducaron todos los derechos y deberes del Estado RDA. Más allá de eso desempeña un rol (11) el hecho de que (independientemente del litigio de si y en qué medida la República Federal de Alemania es sucesor jurídico de la RDA o no) desde hace más de dos décadas la C.E. ya posee competencia político-comercial exclusiva en el ambito de los países miembro. Bajo el carácter confidencial para contratos de derecho civil, la C.E. autorizó que determinados productos pueden ser despachados, libre de arancel y en cantidades tope, desde los antiguos Estados del COMECON ${ }^{(12)}$ o desde Yugoslavia hacia los cinco nuevos estados federales hasta el 31 de diciembre de 1.992. Una precondición para esta liberación arancelaria es (como ya fue mencionado con anterioridad), que los productos correspondientes sean elaborados o consumidos en los cinco nuevos estados federales. Además, se acordó una "escape clause", que puede operar bajo ciertas condiciones.

11) Detalles en: Gilsdorf, P." "Gemeinschaftsrechtiche Probleme im Zusammenhang mit der Abloesung der aussenwirtschaftlichen Verpfligrungen der DDR, en: Zippel, Wulfdiether(ed.): Deutsch-Deutsche Wirtschafts-Waehrungs-und Sozialunion im Rahmen der EG Eaden-Baden. 1.991, p.103 y ss.

12) En la práctica estos contingentes de importación han perdido mucho de su significado. En la medida que los. por aquel entonces, Estados del COMECON habían acordado con anterioridad que a partir del 1 de enero de 1.991 el comercio reciproco debe ser facturado y pagado en moneda convertibie. el intercambio de bienes entre estos paises disminuyó en gran medida. Tan pronto el pago debe facturarse en moneda convertible (es decur. occidental) muchas mercancías procedentes del antiguo biock oriental son bastante poco interesantes en comparación con la oferta en el mercado mundia! 
Otro conjunto de reglas especiales se refiere al empleo de normas técnicas de la C.E. Una parte importante de éstas pudieron ser realizadas de inmediato en los nuevos estados federales. Las restantes normas y prescripciones de seguridad para productos de la C.E. se aplicarán sólo a partir del 1 de enero de 1.993. En relación a las directivas de la C.E. para medicamentos existe incluso un plazo de reorganización hasta el 1 de enero de 1.996.

Un problema especial resulta de la aceptación de los estándares de la C.E. para la protección del medio ambiente. En la ex RDA no existía en efecto legislación alguna sobre el medio ambiente. Es por ello que las emisiones de sustancias perjudiciales por unidad de producto en empresas germano orientales son, en promedio, cuatro y hasta ocho veces más altas que en Europa occidental. Algo semejante ocurre respecto a la calidad del agua potable o en lugares de veraneo. El plazo establecido por la C.E., a partir del cual deben ser observados los estándares de protección ambiental, es en general el 1 de enero de 1.996. Sin embargo, esta concesión rige sólo para instalaciones productivas preexistentes al 3 de octubre de 1.990 .

También se verifican grandes dificultades en la adaptación de la agricultura germano oriental a la Política Agraria Común (PAC) de la C.E. Los costos de producción en la agricultura de la antigua RDA son aproximadamente dos o tres veces más altos que en la C.E. Los precios al consumidor, en cambio, se encuentran significativamente por debajo de aquellos vigentes en Europa occidental como consecuencia de subvenciones estatales muy grandes. Una modificación de esta situación requiere igualmente reglas de adaptación y transición amplias.

Para el sector transporte, la política económica regional (la RDA estaba organizada regionalmente en forma diferente que la C.E.) y para la política de competencia (reglamentaciones especiales, en particular, para la industria del acero y astilleros) rigen prescripciones de transición especiales. En general, aquí se aplican plazos de transición de tres hasta seis años. Visto en conjunto, las reglas de adaptación y transición para la economía germano oriental han sido, tanto por su amplitud como por su duración, considerablemente más rígidas que en el caso de todas las otras ampliaciones de la C.E. realizadas hasta la fecha. Lina porción claramente preponderante de los sectores económicos en los nuevos estados federales está expuesta a la competencia ilimitada desde el resto de la C.E. a partir del 3 de octubre de 1.990 ("shock approach"). No obstante, una cláusula general de flexilbilidad permite a la Comisión de la C.E. modificar las reglas de excepción existentes y formular 
prescripciones para otros ámbitos en caso que esto fuera necesario. En efecto, las diversas actas jurídicas debieron entonces concebirse a velocidad desacostumbrada y sobre la base de informaciones disponibles limitadas.

\section{LOS EFECTOS DE LA REUNIFICACION SOBRE LOS OBJETIVOS MACROECONOMICOS EN LA COMUNIDAD EUROPEA}

El Tratado de la Comunidad Económica Europea (13) (como así también normas jurídicas de los Estados miembro) obligan a la Comunidad (o bien a sus países miembro) a la consecución de cuatro objetivos macroeconómicos, tomando como base principios de economía de mercado. Estos objetivos son el mantenimiento estable del nivel de precios, garantizar un alto nivel de ocupación, un crecimiento económico constante así como mantener el equilibrio económico externo (el llamado "cuadrado mágico").

En este apartado se representa brevemente cuáles son los efectos tendenciales que la reunificación alemana tiene para la consecución de los cuatro objetivos político-económicos superiores de los Estados miembro (o bien para el desarrollo económico de la C.E.). Entretanto, las cuestiones a ello vinculadas pueden ser evaluadas desde un punto de vista cuantitativo considerablemente mejor que en el verano y otoño de 1.990. En aquellas semanas, cuando debieron decidirse las condiciones de la reunificación y de la integracion de Alemania Oriental en la C.E., dominaban considerables déficits de información sobre la real situación económica y social en la ex RDA. Para ello había ante todo tres causas. En primer lugar, la estadística de la RDA acusaba diversas falsificaciones de intensidad desconocida. A ello se agregan problemas metodológicos; mientras que la estadística de producción de los países occidentales se refiere a precios de mercado, en una economía de planificación central imperativa existen en principio precios compulsivos prescriptos por el Estado, los que carecen de aquel valor de información económica. Sin embargo, especialmente problemático, resultó el hecho de no haber podido juzgar de manera aproximada en qué medida las estructuras productivas, que reflejan las preferencias políticas en el ámbito del antiguo COMECON, llegaran a ser competitivas en el mercado mundial.

13) Art. 104 y Art. 2 del Tratado de la Comunidad Económica Europea 
Según la información disponible a comienzos de 1.992 se puede considerar que la productividad media de la mano de obra en la antigua RDA era, aproximadamente, sólo la tercera parte de la correspondiente a Alemania Occidental. Esto significa, que el potencial de producción de los nuevos estados federales en 1.989 representa entre $8 \%$ y $9 \%$ de aquellos de la República Federal de Alemania. Si se refiere al potencial productivo de la C.E., entonces se obtiene un valor de $2 \%$ hasta $2,5 \%$.

La introducción el 1 de julio de 1.990 en Alemania Oriental del DM de convertibilidad ilimitada tuvo como consecuencia -tal como se esperaba- un fuerte aumento de demanda de bienes "occidentales" (14) por parte de ciudadanos germano orientales. Inmediatamente después de la introducción del DM, este aumento de demanda fue financiado prioritariamente (además que con el ingreso corriente) mediante la liberación de una parte de los certificados de ahorro por parte de de los habitantes de la RDA. Poco tiempo después, la capacidad de compra de Alemania Oriental fue estimulada por las significativas transferencias sociales y de ingreso ${ }^{(15)}$, que brindó el gobierno federal para la garantía social del cambio de sistema y para la renovación de la infraestructura muy desgastada de los nuevos estados federales.

La masiva "chupada de importación" de Alemania Oriental, que surgió inmediatamente después de la introducción del DM, significó un impulso adicional para el crecimiento y el empleo para los proveedores. De esto se benefició en primer lugar la antigua Alemania

14) La moneda de la RDA era una moneda puramente interna. Las autoridades impidieron siempre el surgimiento de un mercado negro de divisas eficiente. Esto significa que la economía germanooriental estuvo cuarenta años marginada del desarrollo del mercado mundial. La introducción del DM significó para la economia germano-oriental que desaparecieran de inmediato todas las restricciones de balanza de pagos y, simultáneamente, se abrió un ingreso prácticamente irrestricto a la oferta global de bienes y a los mercados financieros mundiales. De inmediato se pudieron obtener, a precios convenientes, bienes de consumo e inversión extranjeros.

15) A partir de la reunificación, el ingreso disponible de las familias germano orientales proviene, en promedio, en mas que $50 \%$ de pagos de transferencias de Alemania "Occidental"; menos que la mitad del ingreso corriente se genera en la actualidad en el proceso de producción de los nuevos estados federales (la dimensión social de esta ayuda se observa en que los salarios horarios en Alemania Oriental alcanzan actualmente entre $60-65 \%$ (el dia de la reunificación eran aproximadamente $35 \%$ ) de los que son pagados por actividades comparables en Alemania Occidental, aunque la productividad media en los nuevos estados federales alcanza sólo casi el 35\% del nivel germano occidental. Esto modera considerablemente la atractividad para inversores privados y entorpece el proceso de recuperación económica en Alemania Oriental. Por eso se intenta alcanzar, a través de una mejora masiva de las condiciones de inversión en Alemania Oriental (privatización, creación de una infraestructura moderna y actividades de capacitación), que la "brecha de productividad" disminuya tan rapido como sea posible. Hasta entonces, el aumento formal de la productividad se realiza en gran medida mediante una disminución del empleo; sus consecuencias son morigeradas a través de transferencias sociopolítico-financieras desde Alemania "Occidental" 
Occidental, pero también toda la C.E. En última instancia, toda la economía mundial recibe un impulso al crecimiento. La economía germano oriental debe, a diferencia de ello, superar primero grandes problemas estructurales para remontar la "herencia" del socialismo Estos se manifestarán ante todo en un desequilibrio significativo del mercado de trabajo durante varios años. Los antiguos estados federales (i.e. germano occidentales) y la C.E. morigerarán el proceso de adaptación socio político durante el período de la nueva estructuración con voluminosas transferencias financieras. Estos pagos, temporalmente limitados, son desde el punto de vista económico una consecuencia de la rápida introducción del DM y de la pronta incorporación a la C.E. Como consecuencia de ello se ha disminuído efectivamente el número de opciones político-económicas con las cuales se puede proteger temporariamente el proceso de saneamiento de la parte oriental de Alemania. Por ejemplo, ya no es posible mediante la elección de una tasa de cambio adecuada o a través de medidas político-comerciales proteccionistas transitorias (de aplicación degresiva) procurar que la presión competitiva desde el resto de la C.E. se manifieste de forma impulsiva sino paso a paso.

\section{EFECTOS DE LA UNIFICACION ALEMANA SOBRE EL CRECIMIENTO ECONOMICO EN LOS RESTANTES PAISES DE LA COMUNIDAD EUROPEA}

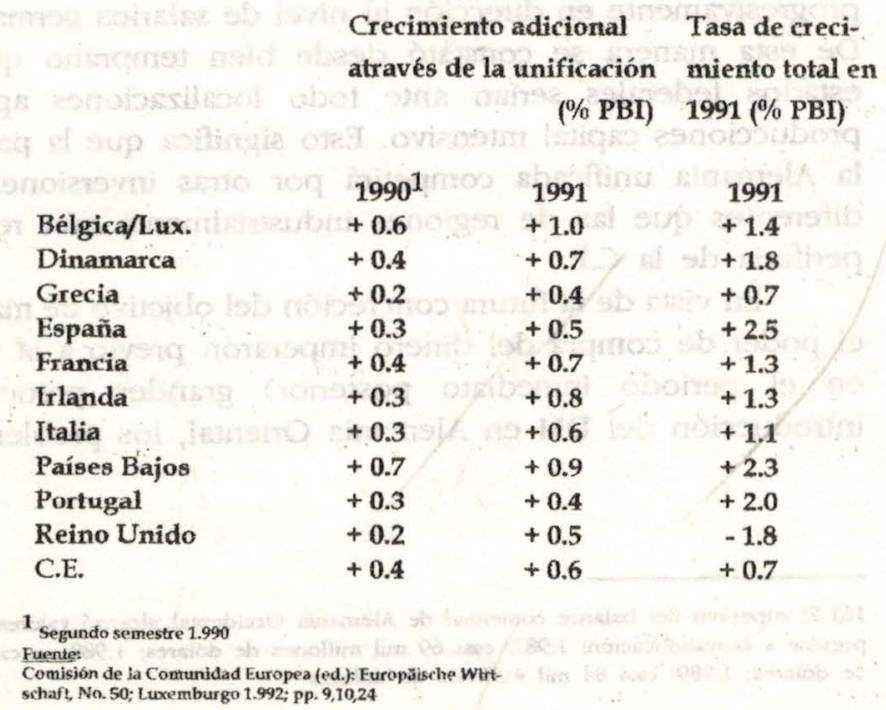


Los efectos sobre el empleo y el crecimiento en beneficio de los socios de Alemania en la C.E. se verifican de dos maneras. El primer camino concieme a ventas directas de bienes y servicios (ejemplo, viajes de vacaciones) a los ciudadanos germano orientales, empresas o instituciones del Estado. Junto a ello, la aceleración del crecimiento en Alemania "occidental" (1.990: casi un crecimiento adicional del 1\% del PNB; $1.991: 1,3 \%-1,5 \%$ ) originada en la reunificación tiene como consecuencia que la demanda de empresas alemanas (occidentales) de materias primas y productos industriales importados desde la C.E. ( $y$ del resto del mundo) se expanda sustancialmente. La financiación de la ola de importación, que directa o indirectamente desencadenó la reunificación, ha sido hasta ahora poco problemática debido a la fortaleza actual de la balanza comercial ${ }^{(16)}$ de Alemania Occidental.

No obstante, los efectos sobre el empleo y la ocupación que acarrearía consigo la reunificación, fueron considerados también con gran preocupación en algunos países de la C.E. En especial, en las regiones económicamente débiles de la C.E. se temió entonces que las empresas de Alemania "occidental" modificarían en lo sucesivo sus planes de inversión en favor de localizaciones en Alemania Oriental. Sin embargo, ya a partir de mediados de 1.990 , tales expectativas se revelaron crecientemente como poco realistas. En efecto, el gobierno federal alemán decidió que los salarios en Alemania Oriental debían convertirse a DM en la relación 1 a 1 y luego ser equiparados progresivamente en dirección al nivel de salarios germano occidental. De esta manera se constató desde bien temprano que los nuevos estados federales serían ante todo localizaciones apropiadas para producciones capital intensivo. Esto significa que la parte oriental de la Alemania unificada competirá por otras inversiones del exterior, diferentes que las de regiones industrialmente aún rezagadas en la periferia de la C.E.

En vista de la futura concreción del objetivo de mantener estable el poder de compra del dinero imperaron previo a la reunifiación (y en el período inmediato posterior) grandes preocupaciones. La introducción del $\mathrm{DM}$ en Alemania Oriental, los problemas de la tasa

16) El superávit del balance comercial de Alemania Occidental alcanzó valores récord en los años previos a la reunificación; 1.987; casi 69 mil millones de dólares: 1.988: cerca de 75 mil millones de dólares; 1.989: casi 84 mil millones de dólares. 
de cambio ${ }^{(17)}$ y los costos exhorbitantes (de magnitud desconocida) de la reconstrucción de la economía germano oriental vastamente arruinada, provocaron el temor de que la tradicional estabilidad del DM pudiera perderse próximamente. Esta preocupación estaba asimismo tan difundida en los restantes Estados de la C.E. como en Alemania. Esto fue así porque la estabilidad del DM es una precondición de importancia decisiva para un desarrollo económico favorable en toda la C.E. En relación a esto se discutió también ante todo el problema de si no cabía esperar en lo sucesivo perturbacio nes funcionales en el Sistema Monetario Europeo (SME) y si, en relación con ello, aparecerán complicaciones en la realización del "Mercado Interno Unico" (i.e. "Single Market Programme"), previsto para el 1 de enero de 1.993.

En aquel entonces había de hecho numerosas razones de peso para tener preocupaciones de este tipo. Tal como fue mencionado, a mediados de 1.990 era imposible estimar con mediana precisión la capacidad de producción que Alemania Oriental tendría en el futuro bajo condiciones de economía de mercado. Con ello faltaba la información de base decisiva para instrumentar sin titubeos una política monetaria orientada potencialmente. Asimismo, en aquel momento nadie podía saber cuál sería la velocidad de circulación del dinero en Alemania Oriental después de la introducción del DM. Más aún, en

17) Salarios y transferencias de ingresos (rentas de pensionados, ayuda a desocupados etc.) fueron convertidos de marcos de la RDA a DM en relación uno a uno. Los certificados de ahorro hasta cierto importe por persona (que fue escalonado según grupos sociales) fueron convertidos asimismo uno a uno; mas allá de este importe, los certificados de ahorro personales fueron acreditados sobre la base de das marcos de la RDA por un DM. Todas otras clases de créditos monetarios y obligaciones fueron convertidos asimismo en relación dos a uno. En promedio para todas las conversiones resulta una relación de intercambio de, aproximadamente, 1,8 a uno.

Según el poder de compra externo, una tasa de cambio realista hubiera sido de aproximadamente 4,5 marcos de la RDA por DM. Pero el empleo de esta relación hubiera conducido a consecuencias sociopolíticas inaceptables. Además, se hubiera desencadenado una emigración masiva de la población de la parte oriental de Alemania. Asimismo, hubieran surgido efectos inaceptables para la función de fondos de estructura de la C.E. En otras palabras: aunque una conversión de salarios y rentas en relación uno a uno no era soportada de ninguna manera por la prochuctividad de la economía germano oriental, no existia ninguna altemativa política realista al tipo de cambio uno a uno como consecuencia de los compromisos extra económicos existentes. El precio económico que se paga por ello consiste en que ahora la mayoría de los puestos de trabajo germano orientales no son rentables (en la medida que produzcan los mismos bienes que antes). Los costos de la financiación de la desocupación a ella asociada y de la mejora de la infraestructura significaron en 1.991 una carga para el presupuesto nacional germano "accidental" de casi $6 \%$ del PNB (el déficit del presupuesto federal a cubrir mediante créditos asciende a casi 4,5\% del PNB). La mayor parte de estas transferencias se emplea en la actualidad para el consumo. Esto explica porque tiene prioridad la mejora de las condiciones de inversión en los nuevos estados federales. Se calcula que serán necesarias transferencias masivas con cargo al presupuesto federal alemán aún hasta el año 2.000 
principio no estaba claro en qué relación los ciudadanos de Alemania Oriental distribuirían su ingreso entre consumo y ahorro bajo estas nuevas condiciones. Además, existía aún una serie adicional de preguntas relevantes, que no podían ser respondidas. También el desconocimiento sobre cómo valorarían los mercados financieros internacionales los múltiples riesgos.

En vista de la compleja problemática sobre qué efectos monetarios tendría la reunificación, la confianza mundial en la tradicional disciplina monetaria del Banco Federal Alemán (Deutsche Bundesbank) se reveló como el factor de estabilización decisivo. Esto fue posible ya que la ley del Banco Federal no se modificó con la introducción del DM en Alemania Oriental. Desde una perspectiva actual se constata que (a causa de la escasa base de información ya descripta) la cantidad de dinero en DM, que entró en circulación el 1 de julio de 1.990, era escasamente el doble de la que entonces hubiera correspondido a las actuales relaciones en el sector de bienes. Mediante el empleo consecuente del instrumental político-monetario el Banco Federal tuvo considerable éxito en "esterilizar" el excedente monetario antes de que pudieran aparecer efectos inflacionarios. Igualmente, existen todavía riesgos inflacionarios latentes. $\mathrm{El}$ principal problema son los altos déficits actuales en el presupuesto alemán, así como el hecho de que éstos se pueden reducir sólo lentamente. En efecto, la reconstrucción económica de Alemania Oriental exigirá aún varios años.

El cuarto objetivo macroeconómico se refiere al equilibrio económico externo. Se mencionó antes que las importaciones de la parte oriental de Alemania aumentaron explosivamente después de la introducción del DM. En función del aumento del ingreso real, las importaciones de los nuevos estados federales crecerán en el futuro más que proporcionalmente. Una expansión equivalente de las exportaciones de Alemania Oriental se alcanzará sólo paulatinamente. Una causa de ello es la baja productividad y la falta de competitividad en precio. A esto se agrega que casi el $70 \%$ de las exportaciones de la ex RDA se destinaban a los Estados del antiguo COMECON. Esta porción del comercio exterior está en quiebra desde 1.991.La situación favorable del comercio exterior de Alemania Occidental ya descripta (ver nota 16) en los años anteriores a la reunificación es una ayuda importante para superar las consecuencias económicas externas de la reunificación. Por lo demás, las importaciones de capital (por ejemplo en forma de inversiones directas del exterior) pueden contribuir, a largo 
plazo, a financiar un déficit del comercio exterior.

Desde el punto de vista de los socios de Alemania en la C.E. (pero también desde la óptica de la economía mundial), la disminución de los excedentes alemanes de comercio exterior fue una contribución muy bienvenida para eliminar los fuertes desequilibrios del balance de pagos de años anteriores (las exportaciones de Francia hacia Alemania aumentaron en 1.991 aproximadamente un 35\%), La reducción de los desequilibrios del balance de pagos es asimismo tendencialmente favorable para la estabilidad de las relaciones de tasa de cambio, acordadas en el marco del Sistema Monetario Europeo. Por lo demás, se puede acudir al gran volumen de facilidades stand-by del Sistema Monetario Europeo y a la estrecha cooperación entre los Bancos Centrales de los países de la C.E. Una desestabilización del SME, como consecuencia de la integración de Alemania Oriental a la C.E., fue entonces desde siempre altamente improbable en la medida en que el Banco Federal Alemán tenga éxito en mantener bajo control la evolución del poder de compra del DM.

\section{LOS EFECTOS SOBRE EL PRESUPUESTO DE LA C.E.}

Al comienzo existió también una gran inseguridad respecto a qué consecuencias tendría la reunificación alemana para la financiación de la C.E. Por cierto estaba claro, que el "empujón financiero" para la introducción del sistema occidental de seguridad social en los nuevos estados federales germano orientales no afectaría directamente ni al presupuesto de la C.E. ni a los presupuestos nacionales de los demás Estados miembro. Por otra parte, no podía existir duda alguna de que los "fondos estructurales" de la C.E. deberían desencadenar pronto gastos adicionales. Asimismo, a mediados de 1.990 se podía evaluar sólo vagamente qué efectos cuantitativos resultarían por el lado de los ingresos del presupuesto de la C.E.

En el caso de los tres así llamados fondos de estructura de la C.E. se trata de "Fondo de Orientación y Garantía para la Agricultura" (art. 40,4 Tratado de la Comunidad Económica Europea); "Fondo Social Europeo" (art. 123 del Tratado de la Comunidad Económica Europea) y el "Fondo Europeo para el Desarrollo Regional" (art.130 c Tratado de la Comunidad Económica Europea). Además, existen diversas formas de las así llamadas ayudas comunitarias, que afectan el lado de los gastos del presupuesto de la C.E. En vinculación con el objetivo de concretar antes del 1 de enero de 1.993 del "Single Market 
Programme", el volumen del "Fondo Regional" se incrementó considerablemente en la segunda mitad de los años '80. La causa de ello fue la intención de garantizar una ayuda especial a las regiones periféricas de la C.E., con lo cual éstas podían prepararse mejor para el mercado interno único.

Este aumento de los recursos del Fondo Regional siempre fue considerado por algunos países miembro como una contribución para la "cohesión" de la C.E. y como "fundamento comercial" para la coincidencia en el "Single Market Programme". Los nuevos estados federales alemanes cumplirían en cualquier caso por lo menos dos ${ }^{(18)}$ de los tres criterios que justifican el acceso a recursos del Fondo Regional de la C.E. El tercer criterio (ingreso medio per cápita en la región a promocionar por debajo del $75 \%$ del promedio de la C.E.) abre la puerta a la mayor parte de los medios del Fondo Regional. Esta precondición no se satisface (más) en los nuevos estados federales alemanes debido a la ya mencionada conversión de los salarios en relación uno a uno. A ello se agrega, que con el propósito de implementar el Fondo Regional, la C.E. ha dividido a los países miembro en "regiones"; en el caso de Alemania Oriental, esta delimitación aún debe realizarse debido a la falta de una base de datos. Este trabajo será concluído recién el 31 de diciembre de 1.993. La Comisión de la C.E. transitoriamente ha puesto a disposición 1.000 millones de $\mathrm{ECU}^{\left({ }^{19)}\right.}$ (aproximadamente U\$S 1.250 millones) por año y durante el período 1.991-1.993 para objetivos de política regional en los nuevos estados federales en block. Con ello, el volumen del Fondo Regional Europeo para los demás socios de la C.E. permanece invariable hasta comienzo del mercado interno común.

En 1.991 habrian fluído hacia la parte oriental de Alemania estimativamente casi 3.000 millones de ECU (cerca de U\$S 3.750 millones) con cargo al Fondo Social Europeo y al Fondo Agrario de la C.E. A ello se agregan los gastos para el sector germano-oriental del carbón y el acero, según criterio del Tratado del Carbón y Acero de la C.E. (20). Además, la Comunidad Atómica Europea ("Euratom") debe contabilizar considerables cargas financieras porque las usinas nucleares de Alemania Oriental deben ser paralizadas por medidas de

\footnotetext{
18) Fomento para regiones económicamente más débiles en la periferia de la Comunidad; ayudas de adaptación para industrias que han dejado de ser competitivas.

19) Miliarden $=1.000$ millones (" "billón" en la terminología americana)

20) Tratado sobre la fundación de la Comunidad Europea para carbón y acero (la llamada "Montanunion").
} 
seguridad. Finalmente, cabe indicar costos de la puesta a disposición de créditos blandos a través del Banco Europeo de Inversión

No obstante, más allá de los gastos adicionales de la Comunidad, la reunificación alemana tiene también un efecto expansivo por el lado de los ingresos del presupuesto de la C.E. En la medida en que, como consecuencia de la reunificación, se importan más bienes desde fuera de la C.E., los aranceles a pagar así resultantes y las quitas en frontera aumentan inmediatamente los ingresos de la C.E. El ingreso medio de la C.E. obtenido de los IVA nacionales ${ }^{(21)}$ aumenta proporcionalmente a la expansión de las transacciones comerciales. Además, cada país miembro está obligado a transferir a "Bruselas", a costo de su presupuesto nacional, el importe necesario para que el aporte nacional alcance anualmente (con imputación de los restantes pagos) 1,2\% de su PNB. Esta última prescripción tiene como consecuencia que en 1.991 Alemania debió girar a la C.E. recursos financieros adicionales por un monto de aproximadamente 2 mil millones de ECU -casi U\$S 2,5 mil millones- (porque el PNB alemán creció de manera explosiva a causa del PNB de los nuevos estados federales).

Sin embargo, el lado de los ingresos del presupuesto de la C.E. crece también porque, como consecuencia de la reunificación, se desató una aceleración del crecimiento en toda la C.E. (en 1.991 casi un $0,7 \%$ adicional). Además, cabe indicar que resultan ciertos ahorros para el presupuesto de la C.E. En efecto, desaparecieron las subvenciones para las regiones aledañas a la antigua frontera interna alemana.

No se dispone aún de datos detallados sobre la magnitud de las implicaciones resultantes para el presupuesto de la C.E. de la integración de Alemania Oriental a la C.E. Mucho habla en favor de que los mayores ingresos al presupuesto de la C.E. alcanzan casi a cubrir los mayores gastos resultantes de la pertenencia a la C.E. de los nuevos estados federales. Este resultado se ha perfilado bastante temprano; ello influenció positivamente la formación de consenso entre los socios de la C.E. para una rápida integración a la C.E. de la parte oriental de Alemania. Además, ayudó políticamente el hecho de que Alemania no haya insistido desde un principio en que se aumente su cuota nacional con la cual está representada en los órganos de la C.E. (la población de la República Federal de Alemania aumentó algo más

21) Actualmente cada país miembro debe destinar anualmente al presupuesto de la C.E. $1,4 \%$ de la base limitada para el IVA. 
que un $25 \%$ a causa de la reunificación). La actitud alemana en esta cuestión es comprensible no sólo por un ahorro de tiempo en la preparación de la reunificación. En efecto, las cuotas de representación serán revisadas de todos modos a más tardar en ocasión de la inminente admisión de nuevos países miembro ${ }^{(22)}$.

\section{EFECTOS PARA LAS RELACIONES EN'TRE LA C.E. Y EL RESTO DEL MUNDO}

Los altos costos sociales y económicos para garantizar la conversión a la economía de mercado de la parte oriental de Alemania, así como los gastos para la creación a tiempo de una infraestructura en la región ingresante, han causado algunas preocupaciones en el resto del mundo. En especial, han surgido dudas de si Alemania y la C.E. podrían verse obligadas en los años ' 90 a reducir sus prestaciones en el marco de la ayuda de desarrollo o su apoyo para Europa central y oriental. Además, se teme a menudo que la C.E. se podría desarrollar como un poderoso imán para inversiones externas directas en desmedro del resto del mundo. Esto dificultaría adicionalmente el problema de endeudamiento de algunos países.

Visto en conjunto, tales preocupaciones parecen cuanto menos exageradas. Es ciertamente correcto, que la situación financiera de la C.E. en los próximos años será tensa por varias razones. Por otra parte, no se puede pasar por alto, que componentes importantes de la relación de intereses económico-mundiales de la C.E. también perdurarán (por ejemplo la dependencia de materias primas minerales importadas y fuentes de energía y las correspondientes necesidades de diversificación). Los efectos dinámicos ya descriptos sobre el crecimiento en los nuevos estados federales alemanes, en Alemania "occidental" y en el resto de la C.E. acarrearán, según todas las experiencias, un aumento más que proporcional de las importaciones fuera de la C.E. De ello se beneficiarán, en primer lugar y de manera especial, los "proveedores" de bienes complementarios (23) Esto significa que las posibilidades de exportación de los países en

22) En la actualidad existen solicitudes de ingreso de los siguientes países: Austria, Suecia, Finlandia, Suiza, Malta, Turquía y Chipre.

23) Desde el punto de vista de la C.E. no se trata de ninguna manera sólo de productos agrarios, materias primas minerales o combustibles; antes bien, los productos trabajo-intensivos (por ejemplo: vestimenta, calzado, artículos del hogar, aparatos electrónicos, maquinarias, juguetes, artículos deportivos etc.) también son considerados (debido a los altos costos salariales y prestaciones sociales) como bienes complementarios. 
desarrollo (LDC) y de los llamados nuevos países industrializados (NIC) mejorarán más que las posibilidades de los países industriales establecidos. Este proceso se puede apoyar de manera efectiva con ayuda del Sistema General de Preferencias Generalizadas (24).

\section{CONCLUSIONES}

La reunificación alemana tiene numerosas implicaciones económicas y extra económicas para el futuro desarrollo de la C.E. Este artículo ha podido tratar sólo algunos aspectos. En vista del propósito de algunos países centro-europeos de ingresar a la C.E. lo más rápidamente posible, se plantea la pregunta de si y en qué medida, las experiencias realizadas en los nuevos estados federales alemanes pueden ser útiles para otros Estados en proceso de reformas fundamentales.

En principio, se constata que la integración a la C.E. del territorio de la antigua RDA representa en muchos aspectos un caso especial y único. Esto se manifiesta, en primer lugar, en la disponibilidad externa de una moneda estable y del marco global de un orden jurídico conforme a una economía de mercado. Las condiciones de partida en los Estados post-comunistas en la frontera oriental de la C.E. se diferencian en esto de manera fundamental. En la actualidad, estos Estados están ocupados en crear un orden jurídico de acuerdo al sistema de mercado y condiciones marco monetarias estables. Los procesos políticos internos de decisión e implementación están más o menos avanzados. La amplia transferencia de recursos que realiza Alemania "occidental" para la cobertura social del proceso de conversión político-institucional y para el saneamiento económico en los nuevos estados federales representa, asimismo, un caso especial irreproducible.

Una experiencia importante para Europa oriental, que actualmente es realizada en los nuevos estados federales alemanes, se refiere al hecho de que las consecuencias económicas de la reintroducción de la propiedad privada se revelan mucho más lentamente que los efectos de las confiscaciones compulsivas realizadas hace más de cuatro décadas. En tanto que las consecuencias personales de la colectivización de la propiedad son incorporadas de inmediato, la reprivatización que acontece muchos años después no provoca sino con cierto rezago

24) Sistema generalizado de preferencias (SGP). 
aquellas reacciones humanas que exige la racionalidad de la economía de mercado. Las formas de pensar y el modo de comportamiento de una economía de mercado deben ser aprendidas por una generación sin experiencias propias en este ámbito mediante un proceso de "prueba y error". El proceso de reforma económica y social se demora así en el tiempo. Análogo vale para el establecimiento de relaciones democráticas estables del estado de derecho. Estas son, asimismo, una condición necesaria para que funcionen a largo plazo los principios del orden de una economía de mercado. La abreviación del proceso de aprendizaje de la economía de mercado se puede favorecer de manera efectiva mediante una transferencia de recursos (materiales y humanos) desde afuera. Por lo tanto, la incorporación del territorio de la antigua RDA en la República Federal de Alemania y en la C.E. se debe designar como un ingreso "de luxe".

\author{
Wulfdiether Zippel \\ Technische Universitaet Muenchen \\ Institut fuer Wirtschafts-und Rechtswissenscbaften \\ Alemania
}

